\title{
The association between maternal sensitivity/availability and attachment in children with autism Spectrum disorder: A systematic review and Meta-analysis
}

\author{
F. Cossette-Côté ${ }^{1} \cdot$ E. L. Bussières ${ }^{2}$ (I) $\cdot$ K. Dubois-Comtois ${ }^{2}$ \\ Accepted: 10 August 2021 / Published online: 2 September 2021 \\ (C) The Author(s), under exclusive licence to Springer Science+Business Media, LLC, part of Springer Nature 2021
}

\begin{abstract}
The association between maternal sensitivity and attachment security has long been established among normative samples. However, less is known about how this association operates among children with Autism Spectrum Disorder (ASD). This meta-analytic review is the first to address the association between maternal sensitivity/availability and attachment security in a population of children with ASD (aged 1 to 7 years) and to explore if this association is moderated by child chronological age, mental age, ASD-related symptoms severity. The objective was also to assess the role of methodological moderators, including the informant of the attachment measure, country and publication year. A systematic search was performed on relevant databases. Seven studies were retained. Meta-analytic results showed a significant medium effect size between maternal sensitivity/ availability and attachment security in children with $\operatorname{ASD}(r=.47 ; 95 \%$ CI: $0.32-.60 ; \mathrm{p}<.001)$, which is a stronger association than in the general population $(r=.24)$. Moderation analyses did not show any significant effect of child chronological age and publication year on effect sizes. The categorical moderators (e.g., informant, country) could not be tested due to the limited number of studies. More research is needed to better understand the way mothers adapt to their children with autism and identify the nuances regarding how maternal sensitivity/availability relates to child attachment in the context of ASD.
\end{abstract}

Keywords Maternal sensitivity; maternal availability $\cdot$ Attachment $\cdot$ Autism spectrum disorder $\cdot$ meta-analysis

Autism is no longer considered a rare condition with a prevalence of more than $1 \%$ (Center for Disease Control and Prevention, , 2012). In Canada, 1.6\% of children aged 8 years are diagnosed with autism spectrum disorder (ASD; Ofner et al., 2018). A similar rate is observed in the United States with a prevalence of $1.9 \%$ ( 1 child out of 54; Maenner et al., 2020). Individuals with ASD show impairments in social interaction and communication, restricted interests and repetitive behaviors present since early childhood (APA, 2013). Social deficits are characterized by a reduction of socioemotional reciprocity, non-verbal behaviors and difficulties in developing, maintaining as well as understanding relationships. These deficits in social interaction and communication

E. L. Bussières

eve-line.bussieres@uqtr.ca

1 Psychology Department, Université de Montréal, 2900 boul Edouard-Montpetit, Montréal, Québec H3T 1J4, Canada

2 Psychology Department, Université du Québec à Trois-Rivières, 3351, Boul des Forges, Trois-Rivières, Québec G8Z 4M3, Canada are likely to influence the quality of parent-child interaction. A small number of studies have looked at parent-infant interactions in the context of ASD in the last decades. Five reviews have synthesized this literature by looking at: 1) parent-child interaction in the context of ASD (Ku et al., 2019) or at-risk of ASD (Wan et al., 2018); and 2) attachment behavior among children with autism (Kahane \& El-Tahir, 2015; Rutgers et al., 2004; Teague et al., 2017).

Specifically, in a meta-analysis including 16 studies looking at parental behavior using only an observational paradigm (parental support/warmth, parental behavioral control, parental negativity and neutral parental behavior), $\mathrm{Ku}$ et al. (2019) did not find any difference between parents of a child with ASD and parents of neurotypical children on two aspects of parental behaviors (parental supportive/warmth and neutral behavior). However, they found parents of children with ASD to be more controlling and negative compared to parents of children without ASD.

Research on parent-infant interaction was also reviewed among infant at-risk of autism for having an older sibling diagnosed with ASD (Wan et al., 2018). Fifteen studies in which parent-child interaction was measured using an 
observational paradigm were included in this systematic review. Results suggest that parent-infant interactions among dyads including a child at risk of autism (and especially an infant who will later be diagnosed with ASD) are different from parent-infant interactions among normative dyads. Their results suggest that children who will later be diagnosed with autism (High Risk-ASD/HR-ASD) interact with their caregiver in a way that depart from a typical trajectory as early as the first year of life. However, parents of HR-ASD showed no difference in interactive behavior from HR-non ASD parents.

These two reviews obtained mixed results regarding parent-infant interaction in the context of autism, suggesting that parents of children with autism tend to interact with their children in a similar way to parents of neurotypical children, with the exception of more pronounced controlling and negative behaviors. However, when looking at child behaviors within parent-child interactions, significant differences are observed between ASD and non-ASD children. These results highlight the need to observe both partners of the dyad to better understand similarities and differences in the parentchild relationship when compared to neurotypical children.

Historically, ASD children were assumed to be unable to develop an attachment relationship with their caregiver (APA, 1980). In their pioneer work, Capps et al. (1994), showed that ASD children were capable of developing a secure attachment with their caregiver (40\% of the sample; Capps et al., 1994). Some other primary studies have been published since, and more recently three reviews have looked at attachment behavior among ASD children (Kahane \& El-Tahir, 2015; Rutgers et al., 2004; Teague et al., 2017).

According to attachment theory, attachment security develops on the basis of repeated experiences of interactions with a mother who is sensitive to her child's physical and emotional needs (Ainsworth et al., 1978; Bowlby, 1969). Numerous empirical studies have confirmed the association between maternal sensitivity and attachment security in neurotypical children from infancy to early school age (e.g., Belsky \& Fearon, 2002; Pederson et al., 1999, 2009; Tarabulsy et al., 2009). A large-scale meta-analysis including sixty-six studies found an association of medium magnitude between caregivers' sensitivity and attachment security in neurotypical children $(r=.24)$ (De Wolff \& van Ijzendoorn, 1997). Considering the social challenges associated with ASD, including the difficulty of signaling and understanding needs, ASD is likely to influence maternal behaviors and subsequent child attachment behaviors.

The first review that addressed the question as to whether children with autism can develop a secure attachment was done by Rutgers and collaborators in 2004. Sixteen studies that included an observational measure of attachment were included in this meta-analysis. The results support the idea that children with autism can show secure attachment behaviors but in a smaller proportion than normative samples ( $53 \%$ vs $65 \%$ ). The authors did not find any moderating effect of chronological age, publication year and matching strategy (matched vs non-matched). Mental age appeared important; in samples including children with autism with a higher mental development, the authors did not find a significant differences in attachment security according to ASD diagnosis. Only in samples with mentally delayed children did the children appear more insecure than their counterparts without autism.

Results from the second review conducted by Kahane and colleagues (Kahane \& El-Tahir, 2015) support those obtained in Rutgers' meta-analysis by concluding that children with ASD can form attachment relationships. However, they found a lower percentage of secure attachment (from 40 to $48 \%$ ) than the medium incidence rate of $53 \%$ found by Rutgers et al. (2004). Some methodological limitations must be acknowledged in this systematic review, including the absence of a critical appraisal of the primary studies included, the heterogeneity of attachment measures and the presence of duplicate samples.

Finally, Teague et al. (2017) performed a wider synthesis of the literature on attachment in ASD children in order to investigate not only attachment classifications, but also the child and caregiving environmental factors that may impact attachment security in children with ASD. Forty studies were included in which child attachment was measured using a variety of techniques, including observations of parent-child interactions, interviews with caregivers, self-report and caregiver-report questionnaires, and symbolic representations. Results confirmed that children with autism are capable of developing attachment behaviors and representations, with $47 \%$ of children categorized as secure $(n=186)$. This is in line with Rutgers' results, but still less than in the general population (60\% categorized as secure; Verhage et al., 2016). The contribution of Teague et al's review is not only to confirm previous results regarding the proportion of secure attachment, but also to add some nuances regarding attachment behaviors of children with autism. It appears that their attachment behaviors are qualitatively different from those of other children, with fewer prosocial responses to the caregiver, deficits in social interaction (e.g., showing, giving, mutual play), more contact resistance, and less contact-seeking behavior, a result also observed in Rutgers' review.

Taken globally, the results of these three reviews suggest that children with autism are capable of developing attachment behaviors toward their caregiver, but the proportion of children categorized as secure is smaller than in the general population. It appears also that the nature of attachment behaviors is qualitatively different from that of normative children. What is not known however, is whether the quality of mother-child interaction predicts ASD children's attachment in the same way as in normative samples. The current study aims at answering this fundamental question. Because 
attachment security has been related to greater social, emotional, cognitive and language abilities in neurotypical children (for an overview, Thompson, 2016) and children with ASD (Bauminger et al., 2010; Rozga et al., 2018), it is relevant to better understand the factors that are related to attachment security in children with ASD. Quality of the motherchild relationship (and particularly maternal sensitivity) has been one of the most studied factors related to attachment in neurotypical children, which has led to multiple meta-analyses over the years (e.g., Atkinson et al., 2000; De Wolff \& van IJzendoorn, 1997; Verhage et al., 2016). Surprisingly, no systematic review and meta-analysis has been conducted over this relationship with ASD children. This is of critical importance to allow a better understanding of how attachment develops in children with ASD not only to advance knowledge on this issue but also to orient services to this population. The objective of this systematic review and meta-analysis is to combine results from the existing literature and to specify the magnitude of the association between maternal sensitivity/availability and attachment security in children with ASD (1-7 years old). This review also aims to address the moderating role of child gender, age, ASD-related symptoms severity, IQ and developmental level on the association between maternal sensitivity/availability and attachment security. We also explore the potential moderating role of methodological variables like publication status, publication year, country in which the study was conducted and the informant of the attachment measure (parent or expert) on the global association.

The present meta-analysis follows the PRISMA Statement reporting guidelines and first presents the methodology of the systematic review (including the literature search and the process of study selection, coding and critical appraisal). The meta-analytic approach and effect size calculation are also presented in this section. The next section presents the results, including the number and characteristics of included studies, the process of critical appraisal, the publication bias analysis, and the meta-analytic results itself (main analysis and moderator analysis). Finally, the implication of results is discussed in the last section.

\section{Methods}

\section{Protocol and Registration}

The protocol of this meta-analysis was not registered.

\section{Eligibility Criteria}

Inclusion Criteria To be retained in this meta-analysis, studies had to include: (1) a sample of children with ASD aged one to seven years; (2) a measuring instrument assessing maternal sensitivity, maternal emotional availability or responsiveness, or mother-child interaction; (3) a measuring instrument assessing attachment security in children; and (4) an association between both measures or relevant information to allow calculation of associations. The studies had to be written in English or in French. No country restrictions were applied.

Exclusion Criteria Studies that included a sample of children at high risk of autism not yet diagnosed, or a sample of children having other neurodevelopmental condition were excluded. Studies in which the mothers had a mental retardation were also excluded.

\section{Literature Search}

Relevant published articles and unpublished theses were searched using two methods. First, systematic searches were conducted using these electronic databases Web of Science, PsychInfo and Medline (Ovid), using the following keywords: (autism* or ASD) AND attachment AND («maternal sensitivity» or «parental sensitivity» or «caregiv* sensitivity» OR «parenting behavior» OR responsiveness). To identify additional literature, thesis summaries were subjected to investigation in ProQuest Dissertations and Theses Global using the same keywords. The research was done in August 2020. No time restriction was applied. The second method used was the screening of the reference lists of all retrieved manuscripts that are included in the meta-analysis (published articles and unpublished thesis) to identify any research that may have been missed through the search engines..

\section{Study Selection}

Study selection was conducted in four specific phases: (a) studies were identified, (b) records were screened, (c) fulltext articles were assessed for eligibility, and (d) studies were included in the review and meta-analysis if they met criteria.

\section{Coding}

The coding of data was done by two authors (F.C.C. \& E.L.B.) who selected the studies according to the inclusion criteria. Two researchers approved the coding grid (K.D.C. $\&$ E.L.B.). The following data were coded: (a) scores of association, mean differences between secure/insecure children in relation to maternal sensitivity/availability $(r, N, M, S D ; p)$; (b) children's chronological age; (c) mental age; (d) sex (\% of boys); (e) attachment measurement (SSP, AQS); (f) attachment informant (mother, expert); (g) mother-child interaction measurement (Maternal Behavior Q-Set -MBQS, MaternalChild Rating Scale- MCRS, Strange Situation-SS, Emotional Availability Scale-EAS); (h) publication year; (i) publication status (published vs. unpublished articles such as theses and 
dissertations); (j) country in which the study was conducted; (k) study design (longitudinal, cross-sectional).

\section{Risk of Bias in Individual Studies}

The quality assessment was performed using the Quality appraisal checklist - quantitative studies reporting correlations and associations (NICE, 2018). The checklist includes 50 key aspects to assess, which are divided into five sections respectively on (1) population; (2) method of selection of exposure (or comparison) group; (3) outcome(s); (4) statistical analyses; and (5) summary. It enables a reviewer to appraise the internal and external validity of each study. The items included in the first section were used to assess the internal validity of the study. Sections 2, 3 and 4 assessed the external validity. All five sections provided an overall quality index for the risk of bias for individual studies. This score, which varied between + and +++ in the original version of the grid, was adapted to provide a numerical score varying between 1 and 3 for each item of the checklist, with 3 being the highest quality score. The scores were then averaged for each section and a total average score was also calculated for each study.

\section{Effect Size Calculation}

Analyses were performed using Comprehensive Metaanalysis 3.0 software (Borenstein et al., 2014). The available statistics provided in the primary studies were entered in their original form into the software and were then converted into the Pearson correlation coefficient $(r)$ since most of the included studies reported a correlation.

\section{Analytic Approach}

An overall effect size was calculated from the effect sizes of the individual studies, with $95 \%$ confidence intervals $(C I)$. The random effects approach was used because of the variability in the methodology used between studies (Borenstein et al., 2011). The attachment measures differed between studies as did the methodology. The random effects approach gives more weight to studies with smaller sizes and less weight to studies with a larger number of participants (Borenstein et al., 2011). Also, this approach presents a wider $C I$ with respect to the coefficient $r$ (meta-analysis) and the slope $\mathrm{b}$ (meta-regression), and is more liberal (Borenstein et al., 2011). A Q-statistic was calculated to verify the heterogeneity between effect sizes, $p<.05$, two-sided (Hedges \& Olkin, 2014). The $\mathrm{I}^{2}$ statistics was also computed to quantify heterogeneity (Higgins \& Thompson, 2002). Finally, continuous moderators were tested through meta-regression. Metaregressions investigate whether specific covariates (moderators) explain any of the heterogeneity of the effect sizes between studies. Separate meta-regressions were conducted for each continuous moderator.

\section{Risk of Bias across Studies}

In order to better establish the validity of our results, we made an effort to minimize the impact of publication bias by searching for unpublished literature (theses and dissertations); publication status and the quality of primary studies were also included as potential methodological moderators in the analyses. Publication bias refers to the tendency that studies reporting higher effect sizes are more likely to be published than studies reporting lower effect sizes. Because published literature is more likely to find its way to a meta-analysis, any bias in the literature is likely to be reflected in the meta-analysis (Borenstein et al., 2005).

In order to estimate more precisely the possibility of publication bias within our data, a funnel plot was created using the CMA software and the Trim-and-Fill Procedure (Duval \& Tweedie, 2000) was performed.

\section{Results}

\section{Study Selection}

The systematic search identified one hundred and fiftyone articles from the research databases. The study selection protocol is fully detailed in a flow diagram (see Fig. 1). Forty-one articles were fully investigated. Four articles were excluded because the sample included children with global delays not specific to ASD. Twelve studies were rejected because they did not include a measure of mother-child relationship and fourteen were excluded because they did not use an attachment measure. Two studies were not primary studies (review) and two studies used the same sample (Koren-Karie et al., 2009; Oppenheim et al., 2012), thus we retained only Koren-Karie et al. (2009). Finally, seven studies were included in this meta-analysis (five published articles and two unpublished theses). The complete list of excluded studies with reasons is available as supplemental material.

The included studies were published between 1994 and 2017 and included a total of 187 participants; samples range from 7 to 48 participants. Two studies included an intervention but only attachment and maternal sensitivity scores that were not influenced by intervention were included in the meta-analysis. In one case, the scores prior to the intervention (pretest values) were used (Deslauriers, 2011) and in the other, considering there were no pretest data, only the scores of the group that did not receive the intervention (non-treated control 


\section{TRISMA PRISMA 2009 Flow Diagram}

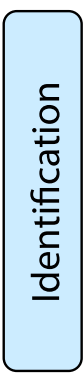

\section{Records identified through \\ database searching}

$(n=151)$
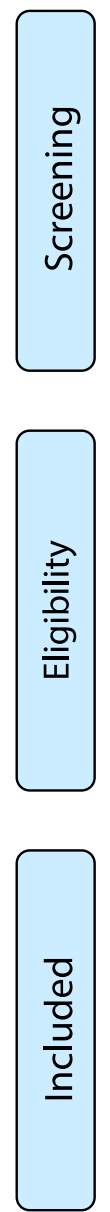

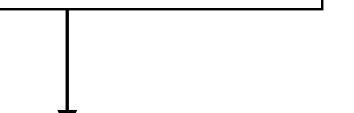

Additional records identified through other sources

$(n=0)$

Records after duplicates removed

$$
(n=127)
$$
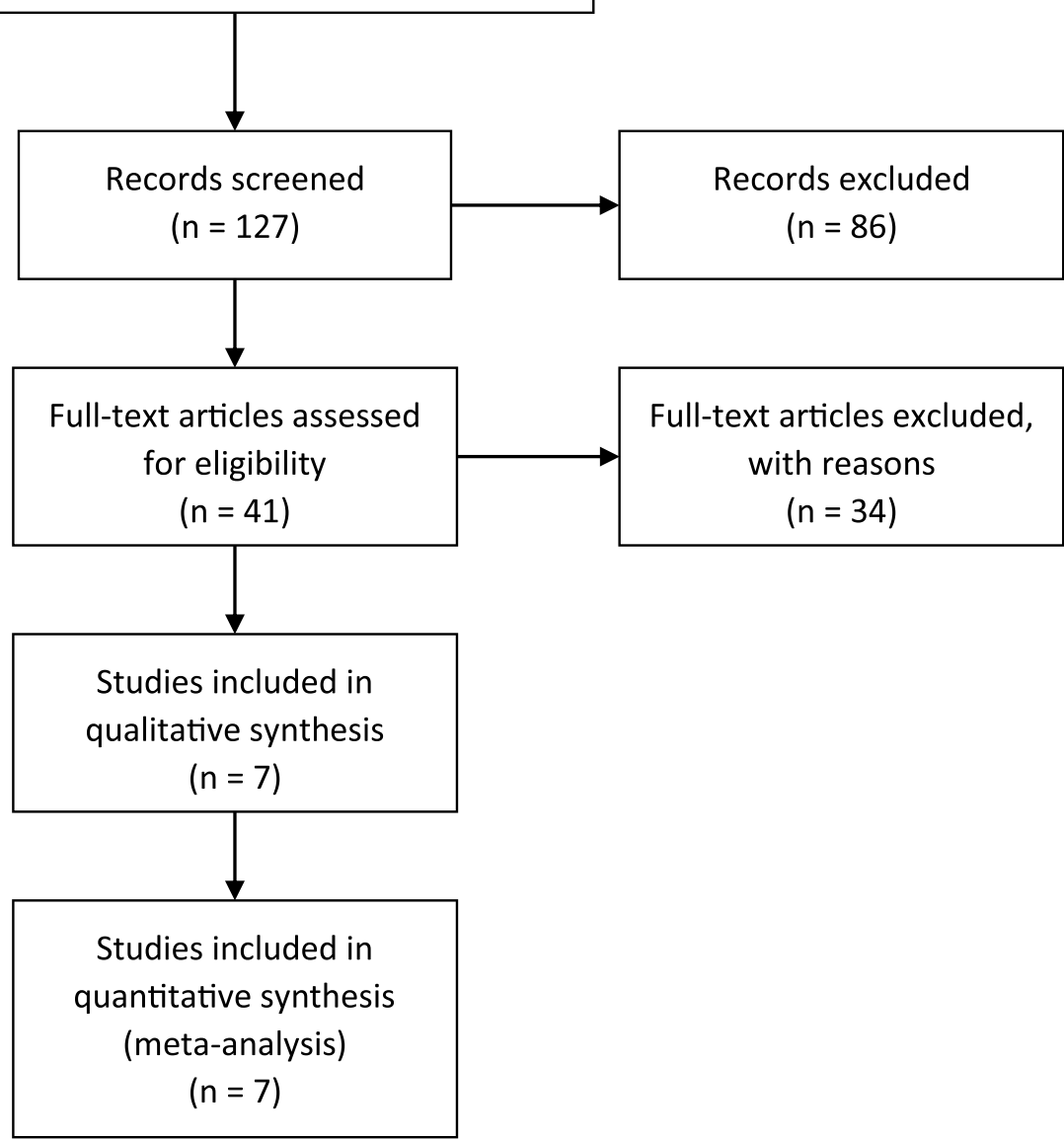

Fig. 1 PRISMA Flow Diagram

group) was extracted (Pechous et al., 2000). Characteristics of the studies are presented in Table 1.

\section{Risk of Bias within Studies}

The quality scores of the studies ranged from 2.50 to 2.73 (Table 2).

\section{Description of Included Studies}

First, we describe the samples and the methods used in the primary studies. In average, mean child age varied from 28.4 to 73 months and most of the children were boys (between 74.5 and $100 \%$ ). Six of the seven studies used an observational measure of attachment (4 used the SSP and 2 the ASQ) and 


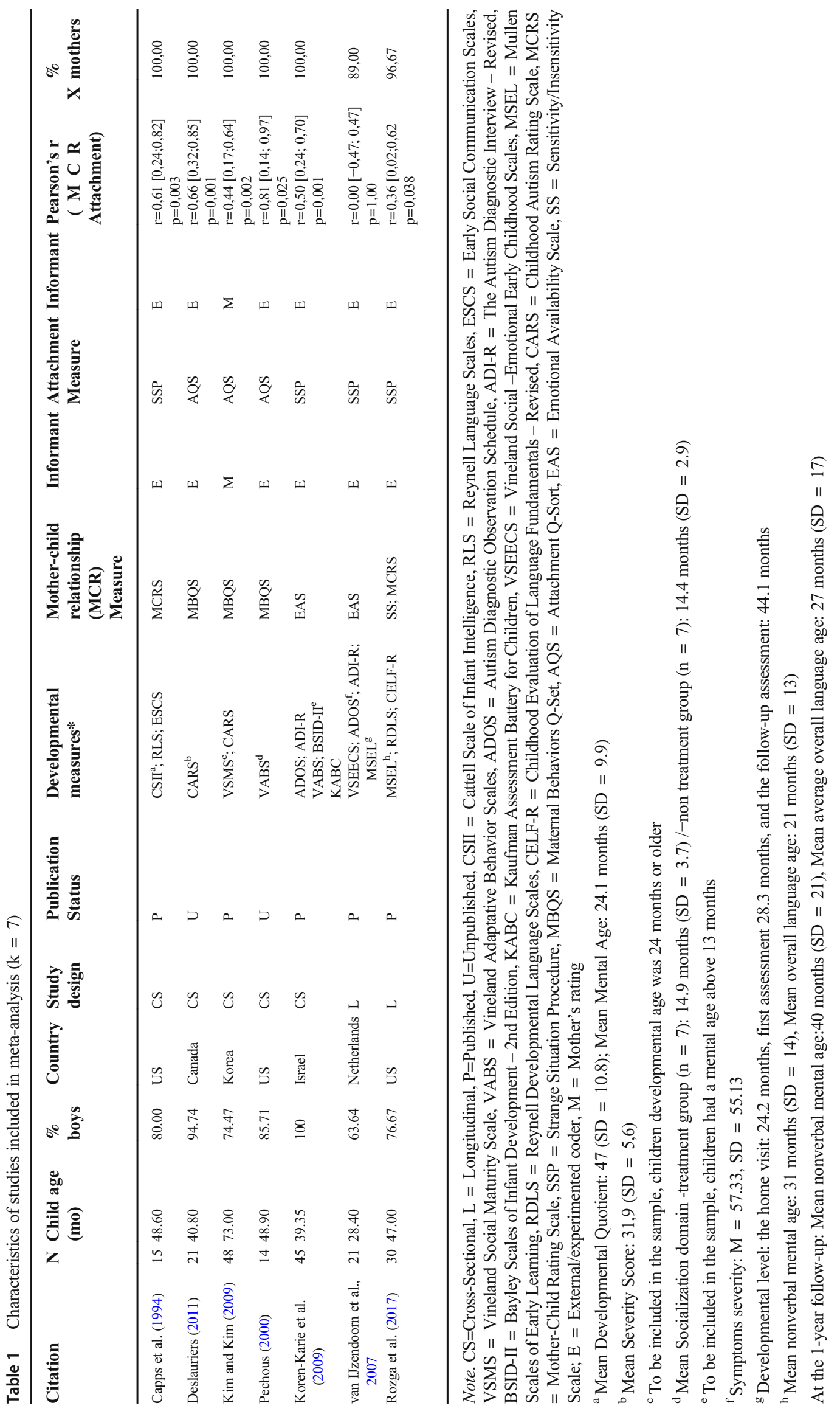


Table 2 Critical appraisal of the studies included in the metaanalysis $(\mathrm{k}=7)$

\begin{tabular}{lccccccc}
\hline & Capps & Deslauriers & Rozga & Kim & $\begin{array}{l}\text { Koren- } \\
\text { Karie }\end{array}$ & Pechous & $\begin{array}{l}\text { Van } \\
\text { IJzendoorn }\end{array}$ \\
\hline $\begin{array}{l}\text { 1. Population } \\
\text { 2. Method of }\end{array}$ & 2.17 & 2.17 & 2.00 & 2.11 & 2.00 & 2.56 & 2.22 \\
$\quad$ selection & 2.50 & 2.25 & 2.67 & 2.25 & 2.50 & 3.00 & 2.80 \\
3. Outcomes & 3.00 & 3.00 & 3.00 & 2.67 & 3.00 & 2.40 & 2.33 \\
4. Analyses & 2.50 & 3.00 & 3.00 & 2.83 & 2.83 & 3.00 & 2.17 \\
5. Summary & 2.50 & 2.75 & 3.00 & 2.50 & 2.50 & 2.50 & 3.00 \\
Total & 2.53 & 2.63 & 2.73 & 2.47 & 2.57 & 2.69 & 2.50 \\
\hline
\end{tabular}

only one study used the mother-report version of the ASQ (Kim \& Kim, 2009). As for the maternal sensitivity/ availability measure, two studies used sensitivity scales (MCRS or SS), three studies used a Q-sort measure of sensitivity (MBQS) and two studies used a measure of emotional availability (EAS); all but one study (Kim \& Kim, 2009) assessed maternal sensitivity/availability by an expert coder. Two studies were unpublished theses (Deslauriers, 2011; Pechous, 2000), both done in North America and using a Qsort instrument to assess both maternal sensitivity (MBQS) and child attachment (AQS); they obtained similar strong correlations between maternal sensitivity and attachment.

Among the seven studies, correlations between maternal sensitivity and attachment varied between .36 and .81 , except van IJzendoorn et al. (2007) who did not find any correlation between the two variables $(r=0)$. These authors found no difference in parental sensitivity between ASD and other groups (mental retardation, language delay, and normative samples), but found a positive correlation between maternal sensitivity and child attachment for every group except ASD children. They used the standard SSP to assess attachment, like the majority of the studies included in the meta-analysis.

Some authors have suggested that an important variable that could act as a possible moderator of the association between maternal sensitivity and attachment behavior of children with ASD is the child's mental age (Rutgers et al., 2004). Others have used language comprehension, level of functioning or severity of ASD as proxy of this construct (Capps et al., 1994; Deslauriers, 2011; Koren-Karie et al., 2009;). Capps et al. (1994) found language comprehension to be higher among securely attached children, but did not find any difference with respect to measures of intelligence. Koren-Karie et al. found no effect of functioning level or children's diagnosis (autism or PDD-NOS) on maternal sensitivity, and Deslauriers et al. found no effect of ASD symptom severity on maternal sensitivity or child attachment.

Some authors found that child social responsiveness played a role; for example, Koren-Karie et al. (2009) showed that child responsiveness was related to maternal sensitivity, but Capps et al. (1994) did not find any difference in social responsiveness among attachment groups.
These mixed results highlight the need for more research on this topic, in order to better understand how maternal sensitivity relates to attachment among dyads including a child with autism.

\section{Main Analysis}

A medium significant effect size was observed between maternal sensitivity/availability and attachment security in children with ASD aged one to seven years $(r=.47 ; 95 \% \mathrm{CI}$ : 0.32-.60; $p<.001$; see Fig. 2).

\section{Publication Bias}

The visual observation of the funnel plot in Fig. 3 shows the presence of a publication bias. An adjusted effect size was calculated using the Trim-and-Fill procedure, indicating an effect size of the same amplitude ( $r=.45[.29 ; .59])$. Therefore, the identified publication bias did not modify the results. No outlier was observed in this meta-analysis.

\section{Additional Analyses}

The Q-test for homogeneity is not significant $(Q=8.27, p=$ .219). Given the fact that small sample size undermines power in meta-analysis, continuous moderators were tested through meta-regression in CMA despite the nonsignificant homogeneity test (Borenstein et al., 2009). The $I^{2}$ statistic was used in complement to the Q statistic in order to quantify the degree of heterogeneity in the aggregated studies, as proposed by Huedo-Medina et al. (2006). The $I^{2}$, which represents the percentage of variation across studies that is due to heterogeneity, was 27.41. More than a quarter of the heterogeneity between studies came from a real difference between studies' effect sizes $(k=7)$. Given the exploratory context of this review and the paucity of literature on the topic, it appeared relevant to perform the moderator analyses in order to explore the role of moderating variables, which could provide further explanations or future directions.

The moderation analyses showed that the percentage of boys included in the samples, child chronological age, quality 


\begin{tabular}{|c|c|c|c|c|c|}
\hline \multirow[t]{2}{*}{ Study name } & \multicolumn{4}{|c|}{ Statistics for each study } & \multirow[b]{2}{*}{ p-Value } \\
\hline & Correlation & $\begin{array}{c}\text { Lower } \\
\text { limit }\end{array}$ & $\begin{array}{c}\text { Upper } \\
\text { limit }\end{array}$ & Z-Value & \\
\hline Capps, L., Sigman, M., \& Mundy. P. (1994). & 0,607 & 0,242 & 0,821 & 3,019 & 0,003 \\
\hline Deslauriers, S. (2011). & 0,660 & 0,319 & 0,850 & 3,364 & 0,001 \\
\hline Kim, E. S., \& Kim, B. S. (2012). & 0,436 & 0,173 & 0,641 & 3,135 & 0,002 \\
\hline Koren-Karie, N. et al. (2009). & 0,500 & 0,235 & 0,696 & 3,474 & 0,001 \\
\hline Pechous, E. A. (2000). & 0,809 & 0,143 & 0,971 & 2,248 & 0,025 \\
\hline Rozga et al. (2017). & 0,356 & 0,020 & 0,620 & 2,072 & 0,038 \\
\hline van IJzendoorn, M. H. et al. (2007). & 0,000 & $-0,467$ & 0,467 & 0,000 & 1,000 \\
\hline & 0,469 & 0,315 & 0,599 & 5,448 & 0,000 \\
\hline
\end{tabular}

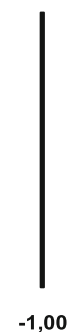

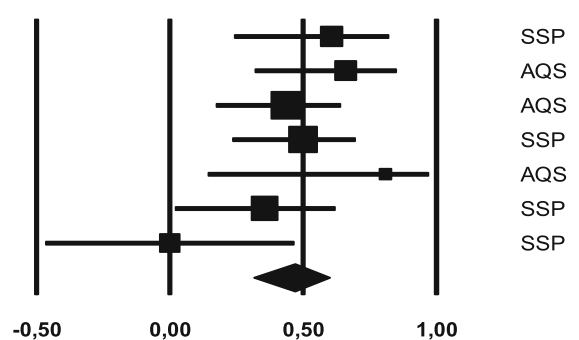

Favours B

\section{Meta Analysis}

Fig. 2 Forest plot

of the studies and publication year did not have a significant effect on the estimated effect size (see meta-regression, Fig. 4, $5,6,7)$. It was not possible to evaluate the impact of child mental age on the association due to lack of information provided in the primary studies. In addition, the categorical moderators (country in which the study was conducted, publication status, ASD severity and the informant) could not be tested, as the number of studies in each category (level of the variable) was less than four (Higgins et al., 2019).

\section{Discussion}

The main objective of this meta-analysis was to determine the magnitude of the association between maternal sensitivity/ availability and attachment in children with ASD aged one to seven years. In this meta-analysis, a significant and medium effect size was found between maternal sensitivity/availability and attachment security in children with ASD, confirming not only that children with autism can develop a sense of security towards the parent, but also that attachment-related behaviors are associated with maternal behaviors including sensitivity and emotional availability. This finding, which was obtained for the first time to our knowledge in a meta-analysis, is important to the field. By confirming that a synchronicity exists between the autistic child and his mother, these results support the relevance of intervention approaches aimed at supporting parental sensitivity.

It appears surprising to obtain a stronger association between maternal sensitivity/availability and attachment security in dyads including a child with autism $(\mathrm{r}=.47)$ in comparison with normative samples ( $r=.24$; see De Wolff \& van Ijzendoorn, 1997 for a meta-analysis). As was suggested by the previous meta-analyses with normative samples, sensitivity plays an important but not exclusive role in the emergence of attachment security. Our results suggest that in samples including children with autism, the role of maternal sensitivity/availability may act differently and play a greater role in the emergence of attachment security. As some authors have mentioned (see Siller \& Sigman, 2002), maternal sensitivity could be especially important in contributing to the development of joint attention in children who have impairments

Fig. 3 Funnel Plot

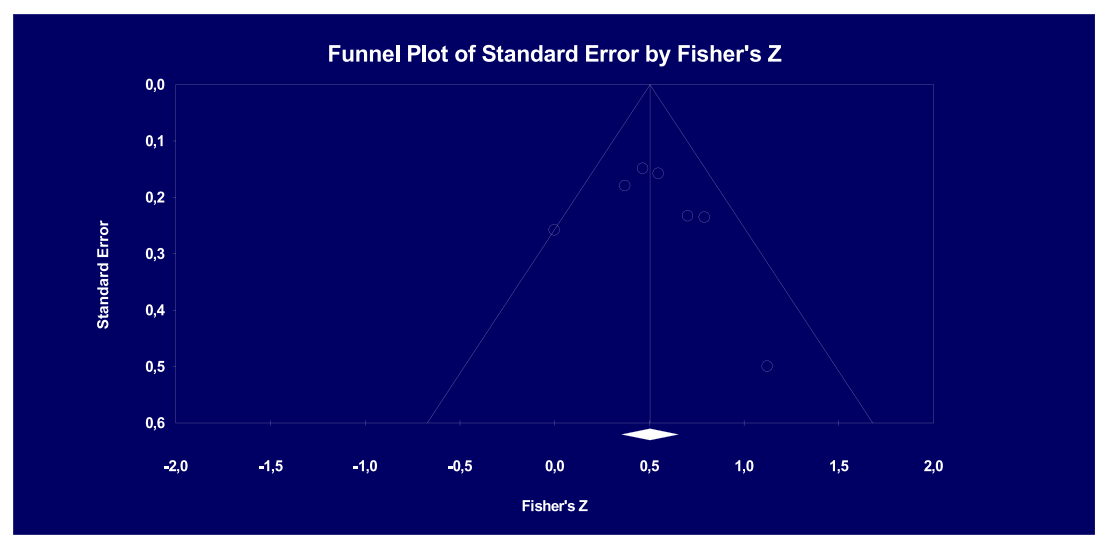


Fig. 4 Meta-regression of publication year
Regression of Year on Fisher's Z

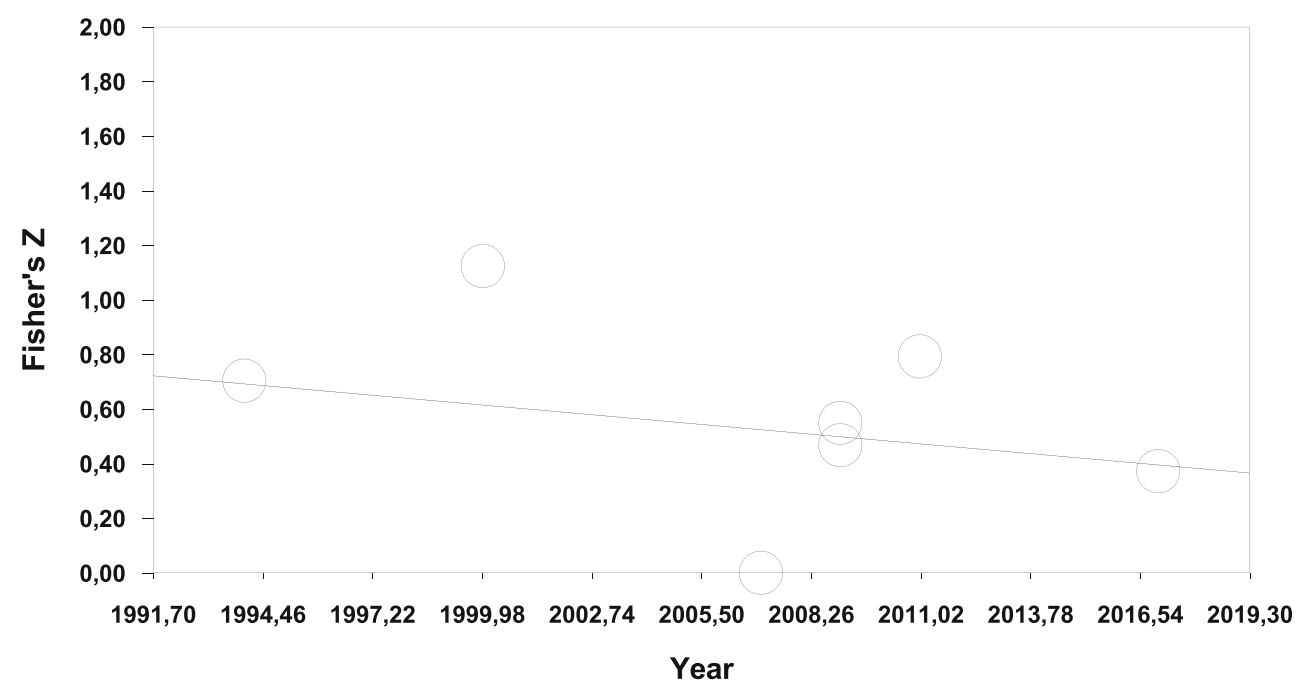

in the use of this ability. Maternal sensitivity may therefore play a greater role in the development of attachment behavior in children with ASD.

Another possible explanation for this stronger association among dyads with ASD is that, following diagnosis, more mothers decide to stay home with their child or work part time to take care of their child with autism than mothers of typically developing children (Cidav et al., 2012). These mothers spend more time with their children and then become their predominant environment. Unlike normative dyads where non-shared environment plays a role in the emergence of attachment (de Wolff \& van Ijzendoorn, 1997), in dyads including a child with autism, it seems that the mother's behavior may play a greater role in the emergence of attachment. This could also be explained by the strong genetic factors implicated in autism; an important proportion of parents of children with ASD present subclinical symptoms, or even a diagnosis of ASD (Constantino \& Todd, 2005). This shared genetic, by reducing the non-shared environment, could be promoting the adoption of similar behaviors between the mother and the child, explaining the moderate correlation between the mother's sensitivity and the child's attachment.

From a methodological perspective, it is also possible that a moderate correlation between sensitivity and attachment indicates close similarity of the constructs and/or the assessment of sensitivity and attachment, rather than an independent association. For example, in studies using self-reported measures for both constructs, it is likely that the correlation observed is the reflection of the same informant's perspective on both variables, rather than a true association between them. This was not an issue in the meta-analysis with neurotypical children, considering child attachment was mostly assessed by

Fig. 5 Meta-regression of age

Regression of Age on Fisher's Z

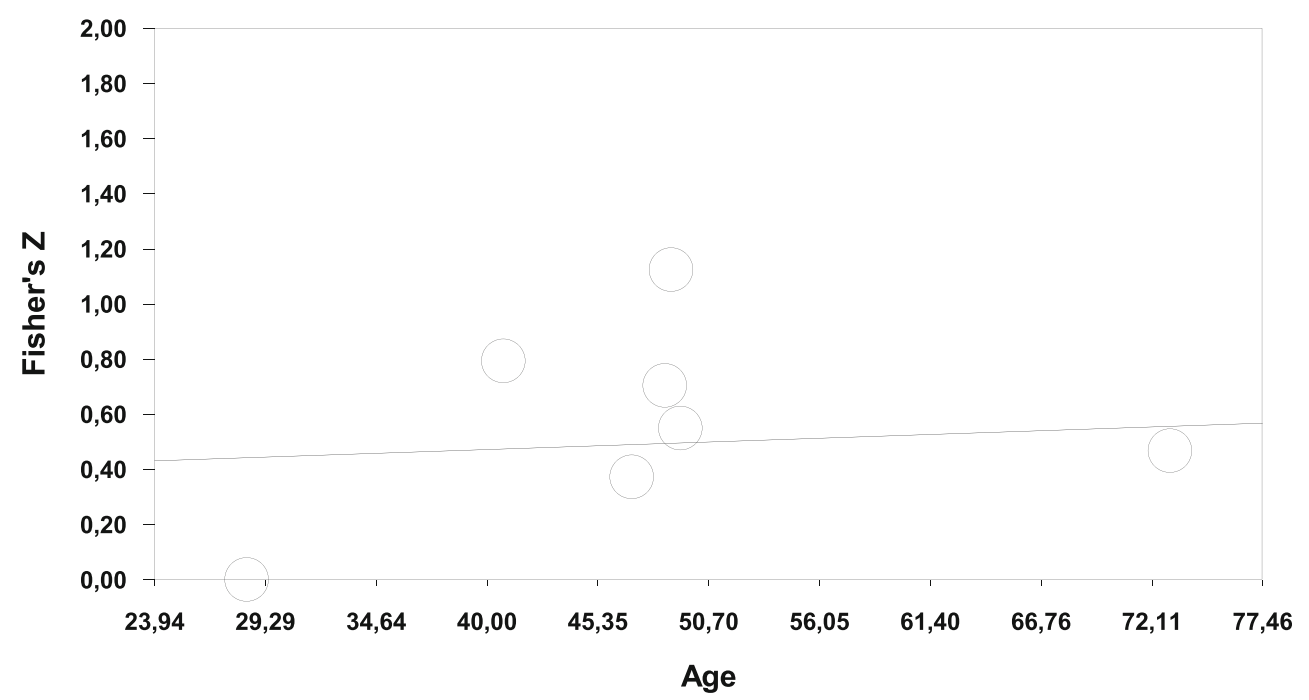


Fig. 6 Meta-regression of $\%$ boys

Regression of \% boys on Fisher's Z

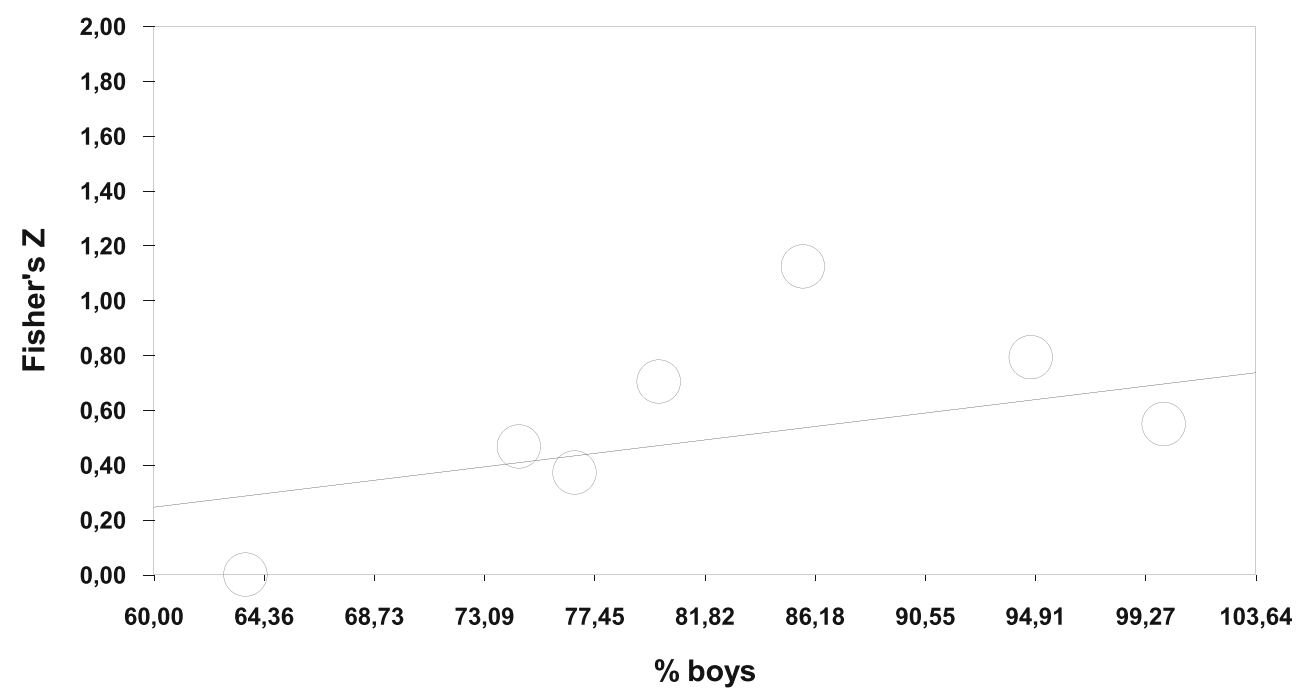

experts during the SSP (Atkinson et al., 2000; De Wolff \& van Ijzendoorn, 1997). It appears as a limit of many primary studies with ASD children where parents are often solicited to assess their child attachment to avoid placing them in a stressful context. Considering that the SSP is a well-validated procedure that allows to assess not only attachment security but also various insecure groups, it should be selected over other attachment measures in future studies with ASD populations.

Regarding the assessment of maternal sensitivity/availability, it remains unclear if the standard measures used by a majority of authors are adequate in the context of autism. As some authors have suggested, our criteria for maternal sensitivity may not apply in the context of ASD (van IJzendoorn et al., 2007). What does being sensitive with a child with autism mean? Is leading the interaction with a child who would not interact otherwise sensitive or intrusive? It is possible that alternative measures would offer a better understanding of the mother-child dynamic, for example, a measure of mother-child interaction, which looks at both partners in the interaction, and therefore takes into account the challenges of interacting with a child who presents social and communicative deficits. However, our results suggest that standard evaluation of maternal sensitivity and attachment can offer us a window into the unique relationship between a mother and her child with autism.

It is clear at this point that more research is needed to better understand the contribution of maternal sensitivity/availability in the development of attachment in these special dyads. The contribution of different moderators also needs to be elucidated, including methodological moderators, as well as variables like mental age and severity of autistic symptomatology. The time spent at home with the child could be an interesting
Fig. 7 Meta-regression of quality scores

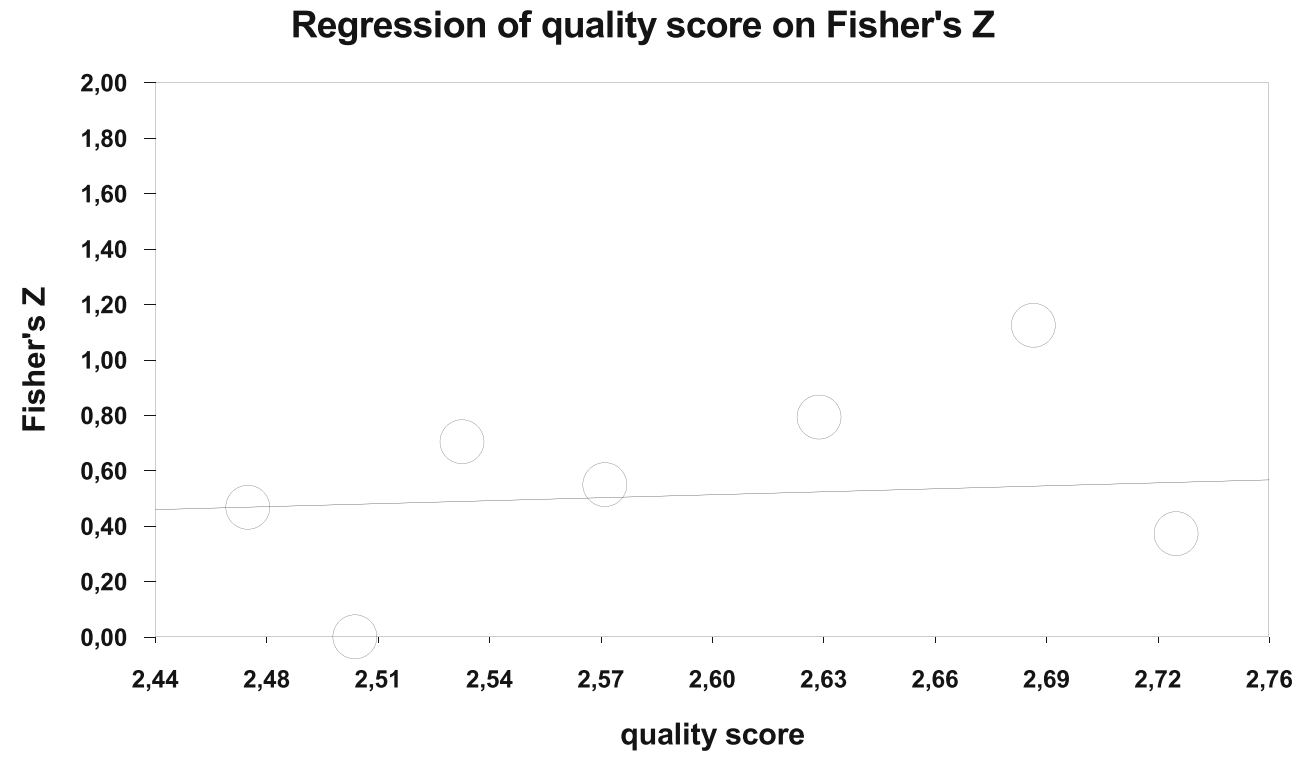


moderator to investigate. The present meta-analysis could not address mental age, severity or autistic symptomatology due to lack of information provided in primary studies and an insufficient number of studies. The moderator analyses conducted for continuous variables did not reveal any significant role of chronological age, gender and publication year on the association between sensitivity and attachment. Rutgers et al. (2004) did not find any moderating role of chronological age either in their meta-analysis. The role of chronological age versus mental age in this association remains to be clarified, although mental age may not be an accurate indicator of functioning in children with autism.

\section{Limitations}

The major limitation of this meta-analysis is the small number of studies included, that made it impossible to perform subgroup categorical analyses and test moderators like the attachment and sensitivity measures and informants, study design and country.

It was also impossible to test the impact of ASD-related symptoms on the association, as the information was not documented in most studies included in this meta-analysis. Although individuals with ASD share several characteristics or common traits, the profiles within the spectrum differ greatly from one individual to another, particularly ASD-related symptoms' severity, level of adaptive functioning, mental age and the presence of associated comorbidities, for example, intellectual impairment.

\section{Future Research}

Future research on the association between maternal sensitivity/availability and attachment among dyads including a child with autism should document children's characteristics like mental age, language abilities, severity of autistic symptomatology, children's responsiveness, as well as the methods used to assess maternal sensitivity and attachment. Different measures of maternal sensitivity or mother-infant interaction should be used in order to capture the unique contribution of maternal behaviors in the context of autism.

\section{Conclusion}

This meta-analysis confirms that the association between maternal sensitivity/availability and attachment exists among dyads including a child with autism, and that this association is twice as strong as in the general population. This finding, which was obtained for the first time to our knowledge in a meta-analysis, is important to the field. By confirming that a synchronicity exists between the autistic child and his mother, these results support the relevance of intervention approaches aimed at supporting parental sensitivity.

This research area being at its early stages, many questions remain unanswered. From a clinical perspective, it is crucial to support these mothers who care for their child with autism every day; their contribution in the emergence of attachment security and later development is crucial and unique.

Supplementary Information The online version contains supplementary material available at https://doi.org/10.1007/s12144-021-02227-z.

Authors' Contributions All authors have made substantial intellectual contributions in the preparation of this manuscript. The first author had the idea for this meta-analysis and performed the literature search, data extraction and critical appraisal under the supervision of E.L. Bussières and K. Dubois-Comtois. The second author (E.L. Bussieres) wrote the article and was responsible for the statistical analysis; consequently, the two first author are considered to have contributed equally to this manuscript.

Funding No funding was received for conducting this study.

Data Availability All data analysed during this meta-analysis are included in this published article [and its supplementary information files].

\section{Declarations}

Ethics Approval not applicable.

Consent to Participate not applicable.

Consent for Publication not applicable.

Conflicts of Interest/Competing Interests The authors report no conflict of interest.

\section{References}

* References marked with an asterisk are included in the metaanalysis

Ainsworth, M. D. S., Blehar, M. C., Waters, E., \& Wall, S. (1978). Patterns of attachment: A psychological study of the strange situation. Lawrence Erlbaum.

American Psychiatric Association. (1980). Diagnostic and statistical manual of mental disorders (3rd ed.).

American Psychiatric Association. (2013). Diagnostic and statistical manual of mental disorders: DSM-5 (5th ed.).

Atkinson, L., Niccols, A., Paglia, A., Coolbear, J., Parker, H. K. C., Poulton, L., et al. (2000). A meta-analysis of time between maternal sensitivity and attachment assessments: Implications for internal working models in infancy/toddlerhood. Journal of Social and Personal Relationships, 17(6), 791-810.

Bauminger, N., Solomon, M., \& Rogers, S. J. (2010). Predicting friendship quality in autism spectrum disorders and typical development. Journal of Autism and Developmental Disorders, 40(6), 751-761.

Belsky, J., \& Fearon, R. M. P. (2002). Early attachment security, subsequent maternal sensitivity, and later child development: Does 
continuity in development depend upon continuity of caregiving? Attachment \& Human Development, 4(3), 361-387. https://doi.org/ 10.1080/14616730210167267

Borenstein, M., Cooper, H., Hedges, L., \& Valentine, J. (2009). Effect sizes for continuous data. In H. Cooper, L. Hedges, \& J. C. Valentine (Eds.), The handbook of research synthesis and metaanalysis ( $2^{\text {nd }}$ ed., pp. 221-235) Russell Sage Foundtion.

Borenstein, M. , Hedges, L. V. , Higgins, J. P. , \& Rothstein, H. R. (2005). Comprehensive Meta analysis software $\left(2^{\text {nd }} \mathrm{Ed}\right)$. Biostat.

Borenstein, M., Hedges, L. V., Higgins, J. P. T., \& Rothstein, H. R. (2011). Introduction to meta-analysis. Wiley.

Borenstein, M., Hedges, L.V., Higgins, J.P.T., \& Rothstein, H.R. (2014). Comprehensive meta-analysis (version 3.3.070) [computer software]. Biostat.

Bowlby, J. (1969). Attachment and loss: Attachment (Vol. I ). Basic books.

*Capps, L., Sigman, M., \& Mundy, P. (1994). Attachment security in children with autism. Development and Psychopathology, 6(2), 249-261. https://doi.org/10.1017/S0954579400004569.

Centers for Disease Control and Prevention. (2012). Prevalence of Autism Spectrum Disorders: Autism and Developmental Disabilities Monitoring Network, 14 Sites, United States, 2008. Morbidity and Mortality Weekly Report. Surveillance Summaries, 61 (3), 1-19).

Cidav, Z., Marcus, S. C., \& Mandell, D. S. (2012). Implications of childhood autism for parental employment and earnings. Pediatrics, 129(4), 617-623. https://doi.org/10.1542/peds.2011-2700

Constantino, J. N., \& Todd, R. D. (2005). Intergenerational transmission of subthreshold autistic traits in the general population. Biological Psychiatry, 57(6), 655-660. https://doi.org/10.1016/j.biopsych. 2004.12.014

*Deslauriers, S. (2011) La contribution de la sensibilité maternelle dans l'établissement d'une relation d'attachement mère-enfant chez les $T E D$ de 24 à 60 mois (Order No. MR88888) [Master dissertation, Université de Sherbrooke]. ProQuest Dissertations \& Theses Global.

De Wolff, M., \& van Ijzendoorn, M. H. (1997). Sensitivity and attachment: A meta-analysis on parental antecedents of infant attachment. Child Development, 68(4), 571-591. https://doi.org/10.2307/ 1132107

Duval, S., \& Tweedie, R. (2000). Trim and fill: A simple funnel-plotbased method of testing and adjusting for publication bias in metaanalysis. Biometrics, 56, 455-463. https://doi.org/10.1111/j.0006341X.2000.00455.x

Hedges, L. V., \& Olkin, I. (2014). Statistical methods for Meta-analysis. Elsevier Science.

Higgins, J. P., \& Thompson, S. G. (2002). Quantifying heterogeneity in a meta-analysis. Statistics in medicine, 21(11), 1539-1558. https:// doi.org/10.1002/sim.1186.

Higgins, J. P., Thomas, J., Chandler, J., Cumpston, M., Li, T., Page, M. J., \& Welch, V. A. (2019). Cochrane handbook for systematic reviews of interventions. John Wiley \& Sons

Huedo-Medina, T., Sanchez-Meca, J., Marin-Martinez, F., \& Botella, J. (2006). Assessing heterogeneity in meta-analysis: Q statistic or $\mathrm{I}^{2}$ index? Psychological Methods, 11(2), 193-206. https://doi.org/10. 1037/1082-989X.11.2.193

Kahane, L., \& El-Tahir, M. (2015). Attachment behavior in children with autistic Spectrum disorders. Advances in Mental Health and Intellectual Disabilities, 9(2), 79-89. https://doi.org/10.1108/ AMHID-06-2014-0026

*Kim, E. S., \& Kim, B. S. (2009). The structural relationships of social support, mother's psychological status, and maternal sensitivity to attachment security in children with disabilities. Asia Pacific education review, 10(4), 561-573. https://doi.org/10.1007/s12564-0099043-y.
"Koren-Karie, N., Oppenheim, D., Dolev, S., \& Yirmiya, N. (2009). Mothers of securely attached children with autism spectrum disorder are more sensitive than mothers of insecurely attached children. Journal of Child Psychology and Psychiatry, 50(5), 643-650. https://doi.org/10.1111/j.1469-7610.2008.02043.x.

Ku, B., Stinson, J. D., \& MacDonald, M. (2019). Parental behavior comparisons between parents of children with autism Spectrum disorder and parents of children without autism Spectrum disorder: A metaanalysis. Journal of Child and Family Studies, 28(6), 1445-1460. https://doi.org/10.1007/s10826-019-01412-w

Maenner, M. J., Shaw, K. A., Baio, J., Washington, A., Patrick, M., DiRienzo, M., Christensen, D. L., Wiggins, L. D., Pettygrove, S., Andrews, J. G., Lopez, M., Hudson, A., Baroud, T., Schwenk, Y., White, T., Rosenberg, C. R., Lee, L. C., Harrington, R. A., Huston, M., et al. (2020). Prevalence of autism spectrum disorder among children aged 8 years - autism and developmental disabilities monitoring network, 11 sites, United States, 2016. Morbidity and mortality weekly report. Surveillance summaries, 69(4), 1-12. https:// doi.org/10.15585/mmwr.ss6904al

National Institute for Health and Care Excellence. (2018). Methods for the development of NICE public health guidance (third edition). Repéré à https://www.nice.org.uk/

Ofner, M., Coles, A., Decou, M. L., Do, M. T., Bienek, A., Snide, J., \& Ugnat, A-M. (2018). Trouble du spectre de l'autisme chez les enfants et les adolescents au Canada 2018: Un rapport du système national de surveillance du trouble du spectre de l'autisme. Agence de santé publique du Canada.

Oppenheim, D., Koren-Karie, N., Dolev, S., \& Yirmiya, N. (2012). Maternal sensitivity mediates the link between maternal insightfulness/resolution and child-mother attachment: The case of children with autism Spectrum disorder. Attachment \& Human Development, 14(6), 567-584. https://doi.org/10.1080/14616734. 2012.727256

"Pechous, E. A. (2000). Young children with autism and intensive behavioral programs: Effects on the primary attachment relationship (order no. 9994805) [doctoral dissertation, California School of Professional Psychology]. ProQuest Dissertations \& Theses Global.

Pederson, D. R., Moran, G., \& Bento, S. (1999). Manual maternal behavior Q-sort version 3.1. Retrieved from http://www.psychology. sunysb .edu/ewaters/349/maternal\%20sensitivity\%20qset.pdf.

Pederson, D. R., Moran, G. S., \& Bento, S. (2009). Assessing maternal sensitivity and the quality of mother-infant interactions using the maternal behaviour Q-Sort (MBQS) [unpublished doctoral dissertation]. University Western Ontario.

*Rozga, A., Hesse, E., Main, M., Duschinsky, R., Beckwith, L., \& Sigman, M. (2017). A short-term longitudinal study of correlates and sequelae of attachment security in autism. Attachment \& Human Development, 1-21. https://doi.org/10.1080/14616734. 2017.1383489, 20.

Rozga, A., Hesse, E., Main, M., Duschinsky, R., Beckwith, L., \& Sigman, M. (2018). A short-term longitudinal study of correlates and sequelae of attachment security in autism. Attachment \& Human Development, 20(2), 160-180.

Rutgers, A. H., Bakermans-Kranenburg, M. J., van Ijzendoorn, M. H., \& van Berckelaer-Onnes, I. A. (2004). Autism and attachment: A meta-analytic review. Journal of Child Psychology and Psychiatry, 45(6), 1123-1134. https://doi.org/10.1111/j.14697610.2004.t01-1-00305.x

Siller, M., \& Sigman, M. (2002). The behaviors of parents of children with autism predict the subsequent development of their children's communication. Journal of Autism and Developmental Disorders, 32(2), 77-89. https://doi.org/10.1023/A:1014884404276

Tarabulsy, G. M., Provost, M. A., Bordeleau, S., Trudel-Fitzgerald, C., Moran, G., Pederson, D. R., Trabelsi, M., Lemelin, J.-P., \& Pierce, T. (2009). Validation of a short version of the maternal behavior Qset applied to a brief video record of mother-infant interaction. Infant 
Behavior \& Development, 32(1), 132-136. https://doi.org/10.1016/ j.infbeh.2008.09.006

Teague, S. J., Gray, K. M., Tonge, B. J., \& Newman, L. K. (2017). Attachment in children with autism spectrum disorder: A systematic review. Research in Autism Spectrum Disorders, 35, 35-50. https:// doi.org/10.1016/j.rasd.2016.12.002

Thompson, R. A. (2016). Early attachment and later development: Reframing the questions. Handbook of attachment: Theory, research, and clinical applications, 3, 330-348.

*van IJzendoorn, M. H., Rutgers, A. H., Bakermans-Kranenburg, M. J., Swinkels, S. H. N., Van Daalen, E., Dietz, C., Naber, F. B. A., Buitelaar, J. K. and Van Engeland, H. (2007). Parental sensitivity and attachment in children with autism spectrum disorder: Comparison with children with mental retardation, with language delays, and with typical development. Child Development, 78(2), 597-608. https://doi.org/10.1111/j.1467-8624.2007.01016.x.

Verhage, M. L., Schuengel, C., Madigan, S., Fearon, R. M. P., Oosterman, M., Cassibba, R., Bakermans-Kranenburg, M. J., \& van IJzendoorn, M. H. (2016). Narrowing the transmission gap: A synthesis of three decades of research on intergenerational transmission of attachment. Psychological Bulletin, 142(4), 337-366. https:// doi.org/10.1037/bul0000038

Wan, M. W., Green, J., \& Scott, J. (2018). A systematic review of parentinfant interaction in infants at risk of autism. Autism, 23(4), 811820. https://doi.org/10.1177/1362361318777484

Publisher's Note Springer Nature remains neutral with regard to jurisdictional claims in published maps and institutional affiliations. 